\title{
Acoustic Coupled Disaster \& Remote Communications Systems
}

\author{
Ulf Müller-Baumgart \\ Serval Project Inc. \\ Paul Gardner-Stephen, \\ Flinders University \\ Andrew Bettison, \\ Flinders University \\ Romana Challans \\ Flinders University \\ Jeremy Lakeman \\ Flinders University
}

Summary: Following disasters, and in other infrastructure-deprived settings, such as remote areas, the need for communications is profound. However, the ability to provide communications solutions in such situations is extremely difficult because of the lack of functional infrastructure, and the logistical difficulty or impossibility of bringing in additional hardware. Therefore it is important to create communications solutions that can operate using existing locally available hardware.

In this paper we describe one possible solution based on acoustic coupling of mobile telephones with existing two-way radios, such as are often carried in remote areas of Australia, and are fitted to many vehicles that are used in such areas.

We describe a number of related software systems and some simple experiments that demonstrate the feasibility of the general concept, before describing a possible integration of such technologies with the secure digital communications capabilities offered by the opensource Serval Mesh software.

We argue that such an integration is not only possible, but also has the potential to allow secure digital communications in a variety of scenarios, without the need for any new or additional hardware.

\section{Introduction}

Disaster situations have many similar properties to peace-time communications in remote areas and developing countries. In each of these three contexts there is a profound need to 
communicate in order to ensure the survival and well-being of people in the area, although the particular dangers and challenges vary.

For example, in a disaster zone there may be a need to communicate the need for assistance when all local communications infrastructure has failed. Similarly, in a remote area, such as the vast expanse of the Australian Outback, there may be a total lack of conventional communications infrastructure (Crouch \& Davies 2013). Finally, in developing countries, there may be a mixture of functional, absent or simply unaffordable communications infrastructure (Yang 2011; Akbari et al 2004; Patricelli et al 2008).

In all three situations, there is desire to enable communications for a variety of purposes. Moreover, in each scenario there are situations where secure communications are required. In a disaster zone this may be to protect the privacy of individual health records, or to enable humanitarian responders to organise without interference from local militia or other parties. The need for privacy also exists in remote areas, including the need to satisfy privacy legislation for medical, economic or other forms of communications. In Australia privacy requirements under law derive primarily from the Privacy Act (Commonwealth) of 1988 (as amended). In international jurisdictions there often exist instruments that similarly impose privacy regulations, although the specifics vary considerably. Finally, in developing countries all of these issues may be present, and in particular, individuals may wish to transfer funds using mobile payment platforms without the opportunity for middle-men to intercept and steal payment codes (Morawczynski \& Miscione 2008; Luo et al 2010).

The challenges to communications in such situations exist in combination with the need for secure communications in these environments. The lack of available, functional, affordable and secure communications infrastructure presents many obstacles.

The Serval Project (Gardner-Stephen et al 2011; 2013a; 2013b; 2013c; 2014) was created to address this challenge, by creating open-source software called the Serval Mesh that can be installed on existing Android smart-phones to allow them to easily carry and disseminate encrypted communications for their owners and for other parties. This application can also be shared from phone to phone, allowing it to be deployed during disasters, in remote areas or developing countries, without recourse to any communications infrastructure.

The primary limitation of the Serval Mesh software is that it is extremely difficult to establish long-range communications between mobile telephones without the use of cellular or Wi-Fi infrastructure. Some solutions are being developed to address this situation, such as the Serval Mesh Extender device (Gardner-Stephen et al 2013b). However, the need for additional hardware does not constitute a viable solution for situations where it is not possible to obtain the hardware in a timely manner, or where the cost would be prohibitive in relation to local 
wages. Therefore there is a need for a solution that can use radio hardware that is already present.

In many of the target situations there will exist some local communications capability in the form of two-way radio, such as HF, VHF or UHF Citizen Band (CB) radio, or its local equivalent. Such systems typically have a range measured in kilometres to tens of kilometres, and thus represent an attractive resource.

From a technical perspective, the challenge is how to make use of an analogue radio system designed for voice communications to easily transfer digital information. At a minimum, it must be possible to automatically modulate and demodulate the communications, so that a human operator is not required to perform this function. That is, human interaction should be limited to, at most, activating the radio transmitter when required.

One approach that is possible and has been used since the 1960s or earlier to interface digital communications systems to analogue bearers is acoustic coupling. Acoustic coupling is simply the creation of a system where the digital system produces an analogue representation, i.e. modulation, of the digital communications and plays it as a sound, which is carried over the analogue bearer. The receiving end of the digital system then listens for such modulated transmission, and demodulates it to recover the digital message (Serrano 1983; Gutzmer 1992). Such MOdulator-DEModulator systems include the dial-up modems common during the early decades of the internet-era (Serrano 1983; Gutzmer 1992). However, it is also possible to use a corresponding mechanism to use two-way radio systems to carry the modulated digital transmissions (Sano 1994).

There are numerous existing technologies for acoustic coupled communication in the amateur radio community. Data transmission is accomplished by using simple baseband modulation schemes in the audio frequency range (eg. AFSK), embodied in software that drives a sound card or speaker, such as the following examples. PSKmail (PSKmail 2014) is a widely known program to send digital encoded messages over the air. The AirChat program (AirChat 2014) by Anonymous uses a similar approach to transmit data. They have all in common the use of Fldigi (Freese et al 2014) as the underlying modulation software. Fldigi provides many more modulation schemes. It has the opportunity to be used as a basis for much more complex modulation systems.

Independent of any particular modulation scheme, our interest is in the ability of a common mobile telephone to perform the modulation and demodulation, using common two-way radio systems, such as the hand-held or vehicle-mounted VHF and UHF CB radio systems found throughout regional and remote Australia. 
The remainder of this paper describes our current thinking on how this might be possible, what candidate radio systems and modulation schemes and software might be leveraged, how these can be integrated with the Serval Mesh, and what the properties of such a combined system might be. We conclude by outlining our plans to realise such a system.

\section{Introduction to Acoustic Coupling}

In the process of acoustic coupling, modulation can be achieved by using common modulation schemes. Usually radio transmission involves modulating the carrier according to some scheme, e.g., frequency shift keying (FSK), where digital signals are represented by modulating different frequencies over the carrier (Usselman 1949). One of the more basic forms of this modulation scheme uses two frequencies to represent the different states of the digital stream. Usually the carrier frequency would be, for example, higher representing a 1 and a bit lower for a 0 .

Audio Frequency Shift Keying (AFSK) is performed by generating an audio signal that encodes the digital information as a series of frequency shifts about a centre frequency (Rappaport 1989; Robertson \& Wood 2005).

For example, one might use a centre frequency of $1000 \mathrm{~Hz}$, which might then be lowered to $800 \mathrm{~Hz}$ to represent a o and increased to $1200 \mathrm{~Hz}$ to represent a 1 . In both cases the duration of the signal is not altered, only the modulation frequency. The centre frequency itself is never used. The resulting audio signal can be transmitted via a normal voice channel like a plain radio. A simple FSK scheme is illustrated in Figure 1.
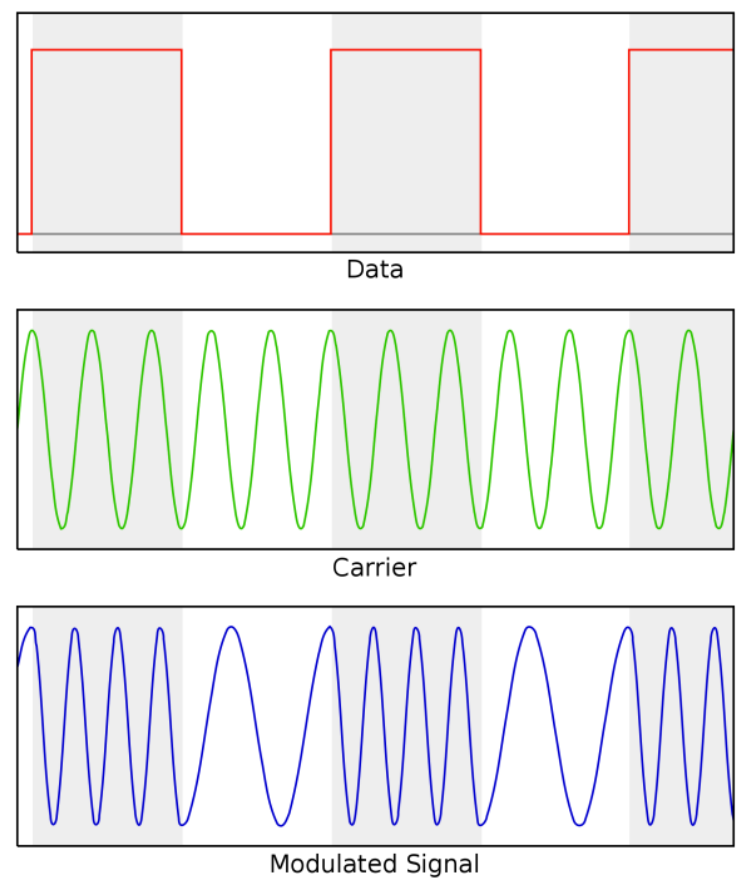
Figure 1 - Modulation by Frequency Shift Keying. Binary digits or logic-levels are represented by different frequencies. In this example a binary 1, represented by high logic level is encoded at a higher frequency (grey background) than a binary 0 , represented by a low logic level (white background) (Tims 2006).

As the voice channel bandwidth is usually limited from $300 \mathrm{~Hz}$ to $3400 \mathrm{~Hz}$ the usable bandwidth is very narrow (Gruen et al 1975, Jayapalan 1987). The modulation method needs to take account of this situation. The limited bandwidth of the channel places an upper bound on the amount of data that can be transmitted per unit time. While the theoretical limit of a simple modulation scheme is around $1.5 \mathrm{~KB} / \mathrm{sec}$, the practical limit tends to be much lower due to a variety of factors (Sklar 2001). To extend the usable throughput, more complex modulation methods, e.g. PSK500 (PSKmail 2014), or QAM (PSKmail 2014) could be used.

\section{Proof-of-Concept Acoustic-Coupled Communications Systems}

To establish the basic feasibility of the central premise of this paper, several simple tests were performed using either a smart-phone speaker to modulate or microphone to demodulate a digital transmission carried by radio.

The software used was PSKmail, which in turn uses fldigi as the underlying software to do the modulation. The modulation scheme is THOR22 (PSKMail 2014). It scheme modulates data at a rate several bytes per second by using a multi tone modulation comparable to MFSK (FSK with multiple frequencies).

In THOR22, the set of multiple frequencies (tones) change over time. This provides resistance to common error sources in radio transmission such as frequency shifts or offsets, e.g., by the Doppler effect or to multi-path propagation. The channel width is a mere $524 \mathrm{~Hz}$, allowing its use on even quite poor radio transmissions paths. The software for THOR22 is available as open source, and therefore presents no licensing barriers to distribution and use.

A preliminary test was performed where the transmitter and receiver were acoustically coupled without a radio path, i.e., were placed next to each other. An Android mobile telephone was used to generate and play the audio-modulated data (Figure 2). This was received without error on another Android mobile telephone (Figure 3) and laptop computer (Figure 4). This confirmed that the modulation-demodulation components were correctly configured and working. 


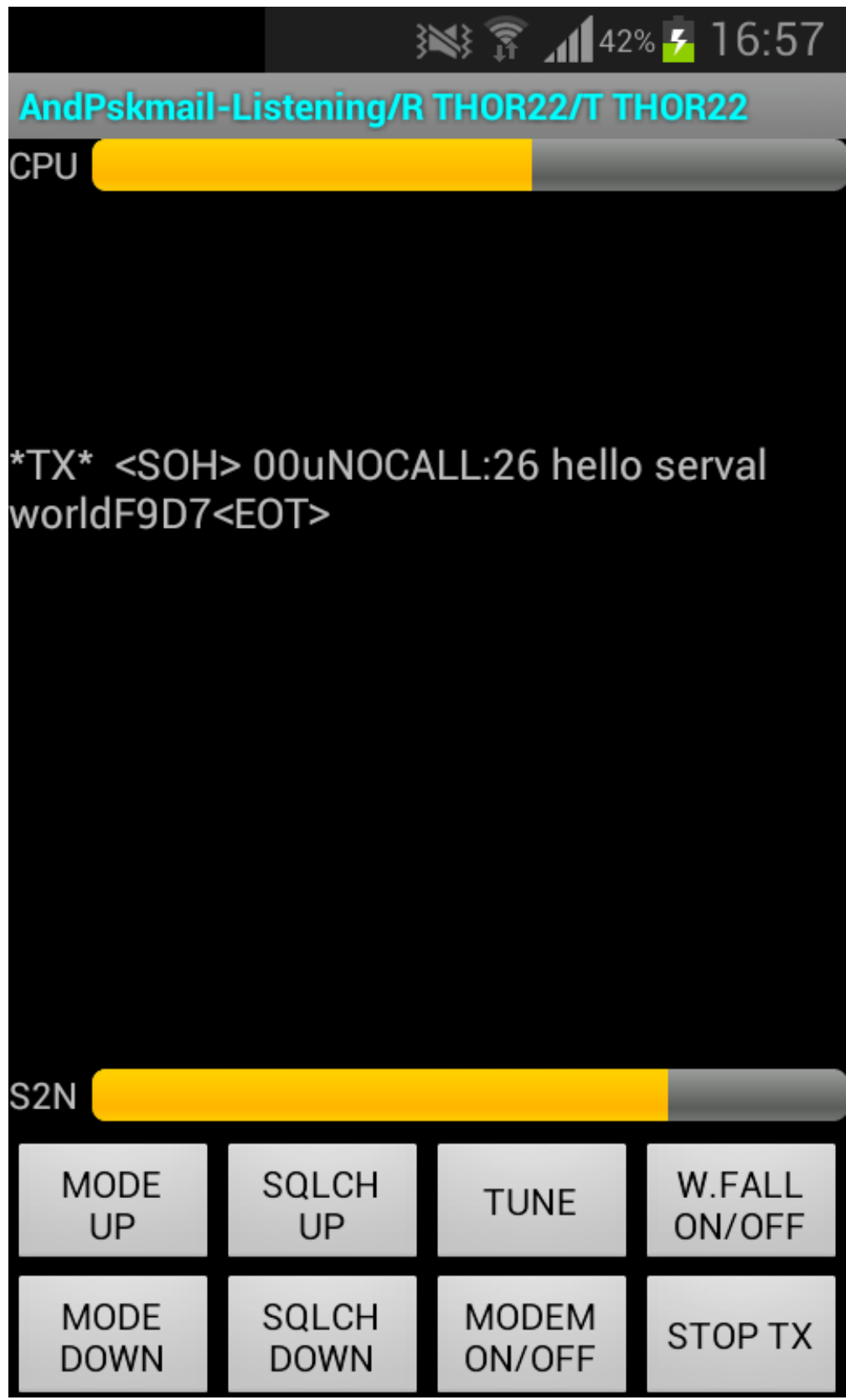

Figure 2 - Android PSKMail program after sending the test message. 


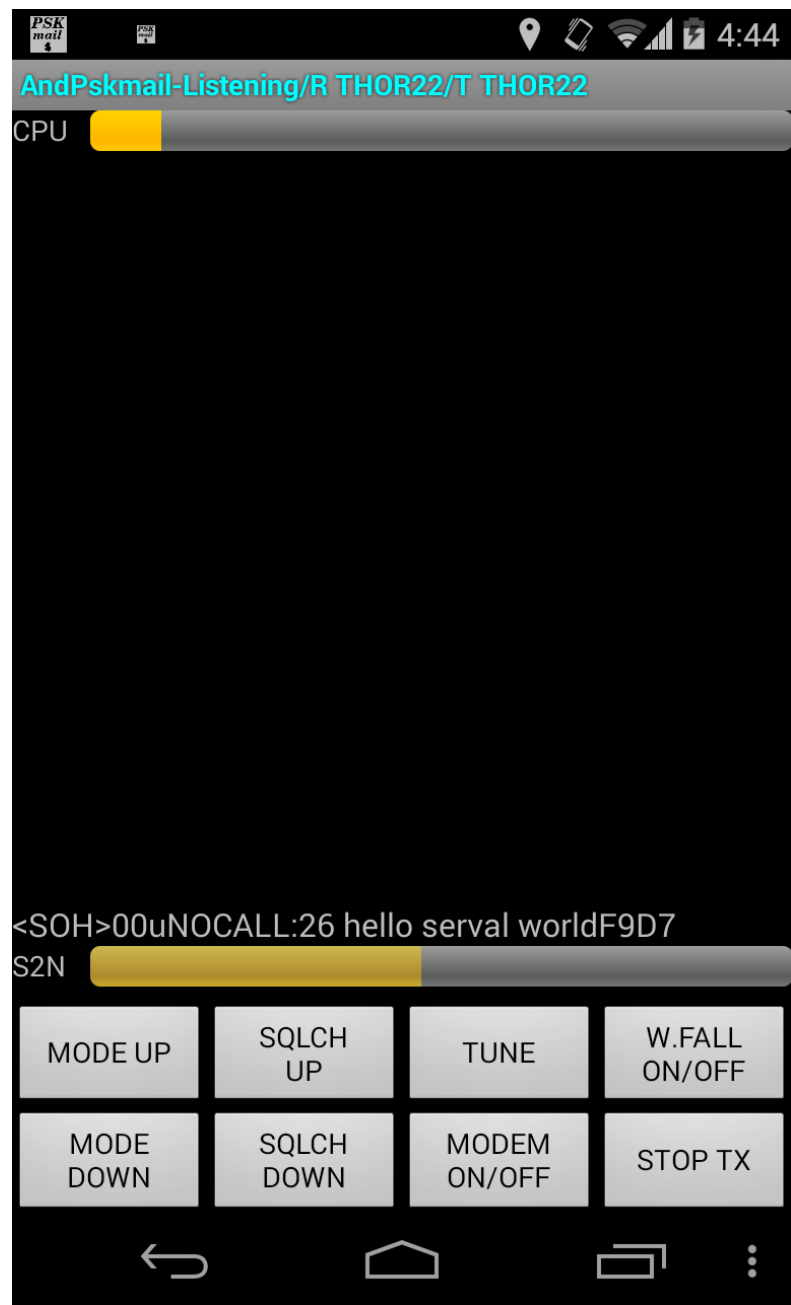

Figure 3 - Android PSKMail program after receiving the test message.

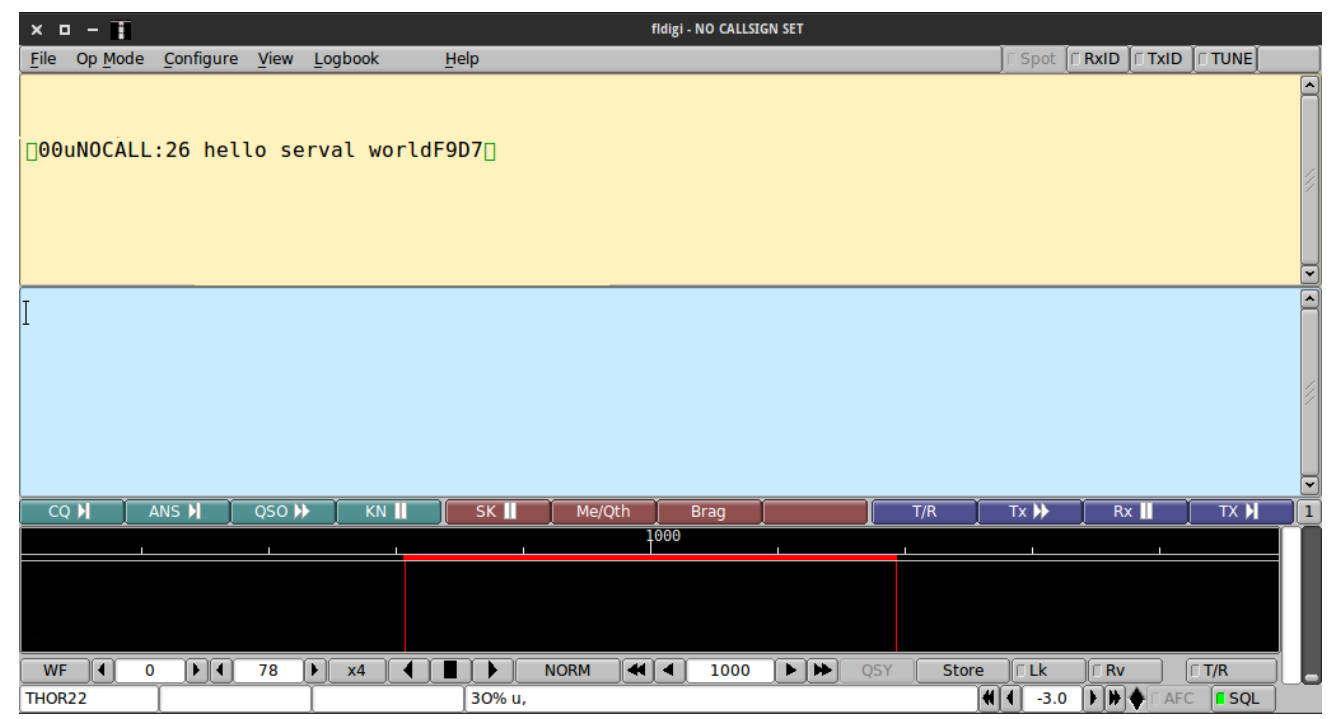

Figure 4 - Laptop version of fldigi after receiving the test message. 
A radio transmission test was done using a car radio and a low-power FM transmitter of the type commonly used to interface MP3 and CD players with older car sound systems. The FM transmitter was a low-cost unit obtained via eBay for less than $\$ 10$, is unlikely to be high fidelity or to have a particularly wide usable bandwidth, and was presumably not designed with digital data transmission in mind. Hand-held CB radio handsets would have also provided an appropriate test platform, however we had no such hardware on hand.

It proved impossible to use the THOR22 modulation in this environment. Some experimentation yielded successful results with the PSK125 modulation scheme, as can be seen in Figure 5 .

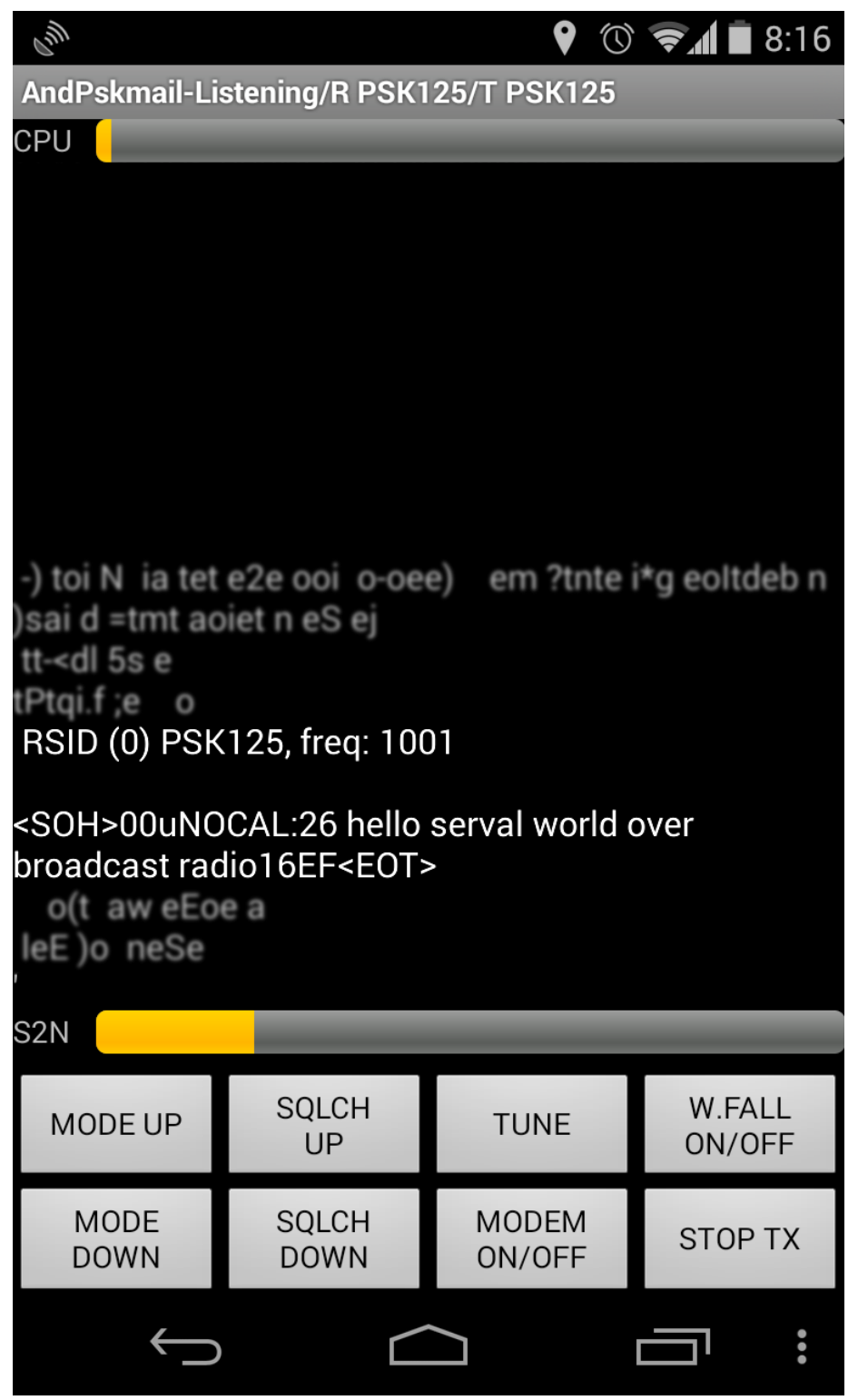

Figure 5 - Mobile phone display after receiving a test message (highlighted) via acoustic coupling over vehicular FM radio. 


\section{Acoustic Coupled Serval Mesh Concept}

From the above discussion it becomes clear that the bandwidth available to an acoustically coupled system will be extremely limited, on the order of $1 \mathrm{~KB}$ per minute. Thus communications will necessarily be limited to extremely succinct forms of communication.

Previous work on the Serval Mesh has focussed on enabling the efficient transmission of text messages and XML forms (Gardner-Stephen et al 2014) via low-bandwidth high-cost satellite links using a purpose written compression library (Gardner-Stephen et al 2013a). This has realised the capability to send useful text messages and compressed digital forms, e.g., of household or personal health assessment, using just a few bytes to several tens of bytes depending on the complexity.

By limiting communications to these compact forms, and allowing for communications overheads, it should be possible to transmit of the order of 10 messages per minute. While such a transmission rate could be easily overwhelmed by a large user-base, in many situations the number of users in a geographic area will be limited, and so the available bandwidth has the potential to support a reasonable number of users. However, to function effectively, it will be necessary to include some mechanism for each participating transmitter to learn and remember which messages have already been transmitted by other stations, so as to minimise redundant transmissions. Similarly, mechanisms already designed into the Serval Mesh to allow deletion of content throughout the network after it has been received will be required. In all cases messages will be transmitted in encrypted form, so that only the intended recipient(s) can decode and read them, unless they are specifically sent out as a public message. Where messages are sent publicly, they will still be cryptographically verified, allowing receivers to have confidence that the message has not been fabricated or tampered with. This allows the possibility of efficiently disseminating information throughout rural and remote communities.

Integrating the acoustic coupled data transport with the existing Serval Mesh protocols makes it possible to share a single UHF or VHF radio among many users, as the Serval Rhizome protocol will allow messages sent from any device to find their way to the nearest radio, and similarly when they have been received by the radio at the far end, to be disseminated to other devices in that area. Thus the acoustically coupled radio transport becomes an addition transport in a Serval Mesh complementing the existing Wi-Fi, cellular, satellite and other transports that are already supported. Likewise, any interconnection between the Serval Mesh and the global communications network can be automatically leveraged in such an integrated scheme. Thus, for example, if someone is seeking emergency assistance, their call for help can 
propagate through all available channels until it is received by a party who can respond, or can be delivered to such a party through contact with the Internet.

By using a radio with VOX activation the transmission would be triggered automatically by the phone by start sending a modulated message. This would give the possibility to provide a fully automated transmission system by simply placing a phone next to a VOX-activated radio at each end.

Figure 6 illustrates this concept by showing a number of mobile telephones running the Serval Mesh software, some of which are acoustically coupled to hand-held or vehicle mounted CB radios. A message entering the system through of these devices will eventually be replicated throughout the system so that the intended recipient can receive it.
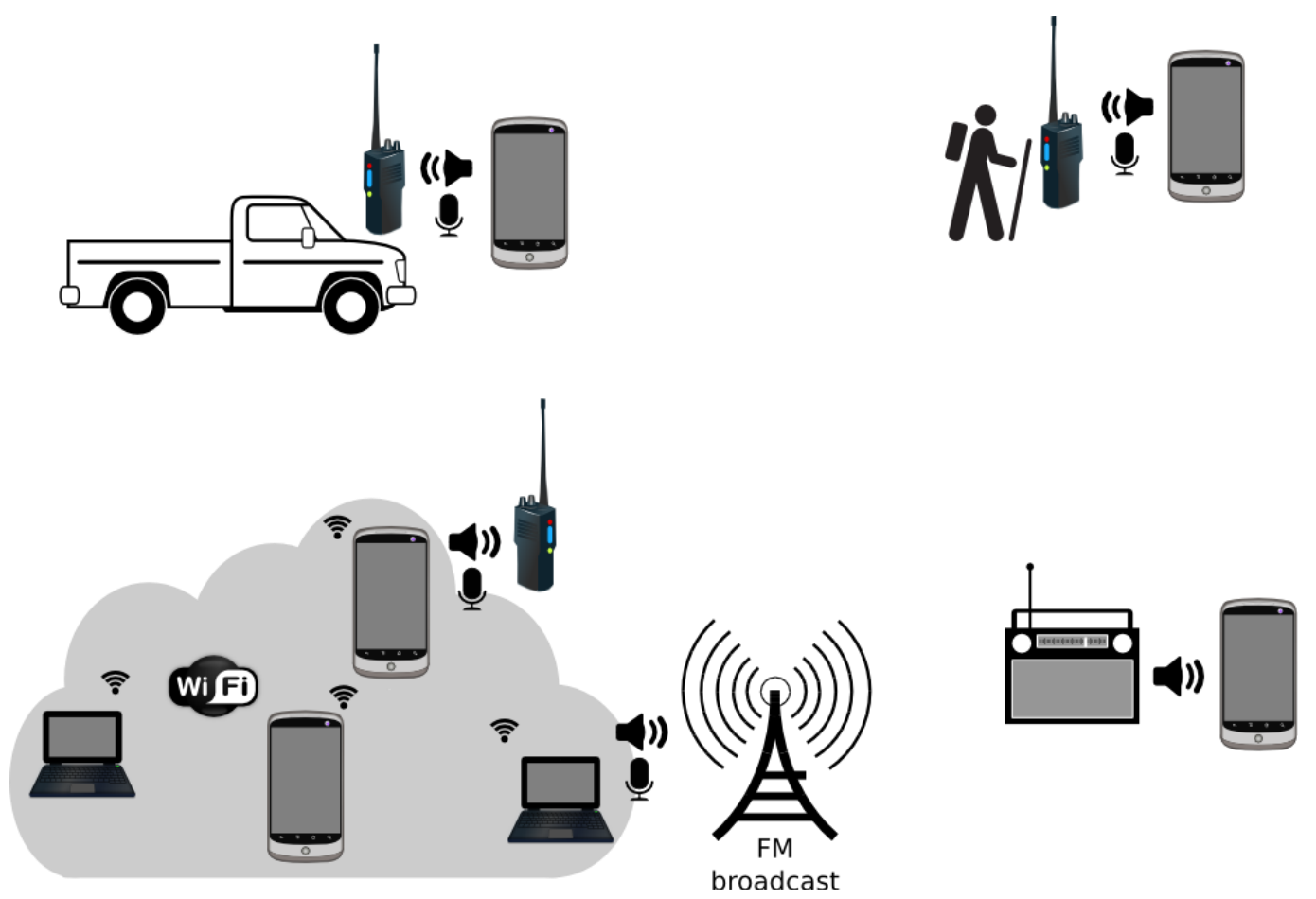

Figure 6 - Potential connectivity options. The cloud in the lower-left represents a local Wi-Fi mesh network. One or more devices in this mesh may host acoustically-coupled transports. These could include broadcast FM, which could provide broadcast of selected data to many devices simultaneously, using common FM radios as the receiver (lower-right in the figure). Alternatively, devices in the mesh could be acoustically coupled to two-way radio units, such as Citizen Band radio. Such transmissions could be received by one or more parties using vehicle-based (upper-left) or hand-held (upper-right) radio receivers.

\section{Anticipated properties, limitations and challenges}

Giving consideration to the opportunities and limitations described above, we anticipate that such a system would be capable of synchronising small amounts of data, including private messages and public announcements among a modest infrastructure-deprived community.

The use of voice-activated (VOX) radios brings the potential for automatic transmission of data. This would require considerable engineering effort to realise in an efficient manner, in 
particular to adapt the Rhizome protocol so that it can manage automatic communications and make effective use of the channel, avoid transmission collisions between devices, and to remember the state of the device(s) that are reachable via radio.

This problem becomes even more complex if there are more than two radios that can transmit on the channel, or if there is no VOX capability in the radio to allow automatic transmission.

The modulation schemes surveyed in this paper all have throughputs that are of the order of kilobytes per minute, and thus will be limited in the data volume that they can be used to communicate per unit time. Considerable work will be required to make use of this limited bandwidth as efficiently as possible. It will almost certainly prove necessary to limit the types of data that will be carried by such media, for example allowing only text messages and other compact data, but excluding large files. The natural broadcast nature of radio transmission does, however, allow the possibility to transmit data such that it can be simultaneously received by multiple stations. This in turn has the potential to render possible the dissemination of general information, such as weather forecasts or the disposition of local services.

There also exist regulatory challenges to this concept, because the class licences for citizen band radio do not necessarily permit digital communications. This requires further exploration.

Finally, some effort would also be required to port the various amateur radio modulation programs to Android and other mobile operating systems, however this carries little technical risk.

\section{Conclusions/Recommendations}

Despite the limitations and challenges of this approach, it seems to the authors that it should be possible to broadcast concise packets of data such as weather information, local news and limited private correspondence in a disaster zone or remote area where there is currently no such possibility. It is primarily in such information vacuums that this capability has the potential to protect life and property against various challenges, and thus derives its value. Therefore the authors intend to explore the realisation of this system as resources permit, with the intention of implementing the necessary protocols and undertaking initial trials in the Australian Outback. 


\section{Acknowledgements}

The Serval Project and authors wish to acknowledge the support of the Flinders University, the NLnet Foundation, the Shuttleworth Foundation, the Open Internet Tools Project, the New America Foundation and other private and institutional supporters.

\section{References}

Akbari, ME; Farshad, AA; Asadi-Lari, M. 2004. The devastation of Bam: an overview of health issues 1 month after the earthquake. Public Health, Volume 118 , Issue 6 , 403 408

Crouch, A; Davies, S. 2013. A coordinated satellite and terrestrial microwave backhaul for cellular mobile in remote and regional Australia [online]. Australian Journal of Telecommunications and the Digital Economy, Vol. 1, No. 1, Nov 2013: [2.1]-[2.19]. Availability: http://search.informit.com.au/documentSummary; $\mathrm{dn}=776009909539470 ;$ res=IELBUS SSN: 2203-1693. [cited 12 Dec 14].

Freese, D. et al. 2014. Fldigi. Available at: http://www.w1hkj.com/Fldigi.html, retrieved 13 Oct 2014.

Gardner-Stephen, P; Bettison, A; Challans, R; Hampton, J; Lakeman, J; Wallis, C. $2013 \mathrm{a}$. "Improving Compression of Short Messages." Int'l J. of Communications, Network and System Sciences 2013.

Gardner-Stephen, P; Caldicott, E; Challans, R; Lakeman, J; Bettison, A; Gardner-Stephen, D; Lloyd, M; Malossane, T; Wallis, C. 2014. "Succinct Data: Extreme Compression for ODK Forms." To appear in Global Humanitarian Technology Conference (GHTC), 2014 IEEE.

Gardner-Stephen, P; Challans, R; Lakeman, J; Bettison, A; Gardner-Stephen, D; Lloyd, M. 2013b. "The serval mesh: A platform for resilient communications in disaster \& crisis." In Global Humanitarian Technology Conference (GHTC), 2013 IEEE, pp. 162-166. IEEE.

Gardner-Stephen, P. 2011."The Serval Project: Practical Wireless Ad-Hoc Mobile Telecommunications." Available at: http://developer.servalproject.org/files/CWN Chapter Serval.pdf, retrieved Oct 13, 2014 .

Gardner-Stephen, Paul; Bettison, Andrew; Challans, Romana; Lakeman, Jeremy. 2013c. "The Rational Behind The Serval Network Layer For Resilient Communications." Journal of Computer Science 9, no. 12 (2013): 1680.

Gruen, H; Divita, PS; Werneth, CJ. 1975. "Variable Bandwidth Voice and Data Telephone Communication System." U.S. Patent 3,875,339, issued April 1, 1975.

Gutzmer, HA. 1992. "Acoustic coupler for high-speed modem." U.S. Patent 5,134,649, issued July 28, 1992.

Jayapalan, JP; Lu, CC; Miller, WJ; Stephenson, JE. "Digital voice transmission having improved echo suppression." U.S. Patent 4,652,703, issued March 24, 1987.

Luo, X; Li,H; Zhang,J; Shim, JP. 2010. "Examining multi-dimensional trust and multifaceted risk in initial acceptance of emerging technologies: An empirical study of mobile banking services." Decision Support Systems 49, no. 2 (2010): 222-234. 
Morawczynski, O; Miscione, G. 2008. "Examining trust in mobile banking transactions: The case of M-PESA in Kenya." In Social Dimensions of Information and Communication Technology Policy, pp. 287-298. Springer US, 2008.

Patricelli, F; Beakley, JE; Carnevale, A; Tarabochia, M; Von Lubitz, Dag KJE. 2009. "Disaster management and mitigation: the telecommunications infrastructure" Disasters Volume 33, Issue 1, pp 23-37. Available at: http://onlinelibrary.wiley.com/doi/10.1111/disa.2008.33.issue-1/issuetoc

Rappaport, TS. 1989. "Indoor radio communications for factories of the future." IEEE Communications Magazine, 27, no. 5 (1989): 15-24.

Robertson, B; Wood, T. 2005. "Audio FSK encoded digital data packet embedded in the audio message portion of an EAS protocol transmission, and related methods." U.S. Patent Application 11/o91,735, filed March 28, 2005.

Sano, H. 1994. "Method and arrangement of echo elimination in digital telecommunications system." U.S. Patent 5,313,498, issued May 17, 1994.

Serrano, AL. 1983. "Modem telephone interface circuit." U.S. Patent 4,367,374, issued January 4, 1983.

Sklar, B. 2011. Digital communications. Vol. 2. NJ: Prentice Hall, 2001.

Tims, K. 2006. "An example demonstrating Binary Frequency-shift Keying." http://commons.wikimedia.org/wiki/File:Fsk.svg, retrieved Dec 12, 2014.

Usselman, GL. 1949. "Frequency shift keying." U.S. Patent 2,461,456, issued February 8, 1949.

Airchat. 2014. Various contributors.. AirChat. Available at: https://github.com/lulzlabs/AirChat, retrieved 13 Oct 2014.

PSKMail. 2014. Various contributors. 2014b. Available at: http://pskmail.org, retrieved 13 Oct 2014.

Yang Ran. 2011 "Considerations and suggestions on improvement of communication network disaster countermeasures after the Wenchuan earthquake," Communications Magazine, IEEE, vol.49, no.1, pp.44,47, January 2011 doi: 10.1109/MCOM.2011.5681013 Available at:

http://ieeexplore.ieee.org/stamp/stamp.jsp?tp $=$ \&arnumber $=5681013$ \&isnumber $=56810$ $\underline{03}$ 